\title{
Reptonoditrypa cautica, briozoo incrustante de los olistolitos Cipit de la Formación San Cassiano (Triásico, Ladiniano/Carniano; NE de Italia) y sus implicaciones paleoecológicas
}

\author{
Francisco Sánchez-Beristain, Joachim Reitner
}

Francisco Sánchez-Beristain sanchez@ciencias.unam.mx

Museo de Paleontología, Facultad de Ciencias, Universidad Nacional Autónoma de México, Av. Universidad 3000, Coyoacán 04510, CDMX.

\section{Joachim Reitner}

Centro de Geociencias de la Universidad de Göttingen, Goldschmidtstraße 3, D-37077 Göttingen, Alemania.

\section{RESUMEN}

Reptonoditrypa cautica Schäfer y Fois es una especie de briozoo que formó parte importante del gremio de los unificadores/incrustantes en los arrecifes del Triásico, principalmente en los pisos Ladiniano y Carniano. En los olistolitos Cipit de la Formación San Cassiano se encuentra normalmente asociado a ejemplares de la esponja talámida Cryptocoelia zitteli (miembro del gremio de los constructores/amortiguadores), aunque también puede formar costras en conjunto con carbonatos microbianos sobre otras especies de constructores de arrecifes. Es de destacar que, si bien la esponja siempre aparece ligada al briozoo en las láminas observadas, la presencia del mismo no depende aparentemente de la propia esponja. Resalta asimismo la ausencia de Reptonoditrypa cautica en otras muestras de facies arrecifales en lámina delgada provenientes de dichos olistolitos, además de ser la única especie de briozoo microencostrante encontrada en cerca de 400 láminas delgadas.

En este artículo se explica a detalle el rol paleoecológico que esta especie desempeñó como parte de los sistemas arrecifales de la Formación San Cassiano.

Palabras clave: Briozoos, incrustantes, gremios arrecifales, Triásico, Formación San Cassiano.

\section{ABSTRACT}

$\underline{\text { Reptonoditrypa cautica Schäfer and Fois }}$ is a bryozoan species that constituted an important part of the encrusting/binder guild of the Triassic reefs, mainly in the Ladinian and Carnian. In the Cipit boulders of the St. Cassian Formation, it is usually associated with specimens of the thalamid sponge Cryptocoelia zitteli (a member of the constructor/baffler guild), although it can also be seen forming crusts along with microbialites on top of other species of reef builders. It is worth mentioning that, although the sponge is always linked to the bryozoan, this does not apply for the opposite. It is also worth to pinpoint the absence of Reptonoditrypa cautica in other thin-section samples of reef facies from these boulders, as well as the fact that it is the only kind of microencrusting bryozoan found in about 400 thin sections.

This article explains the paleoecological role played by this species in the reef systems of the St. Cassian Formation.

Keywords: Bryozoans, encrusters, reef guilds, Triassic, St. Cassian Formation.
BOL SOC. GEOL MEX 2017

VOL. 69 NO. 2

P. $409-420$

Manuscrito recibido: Diciembre 13, 2016.

Manuscrito corregido: Enero 13, 2017.

Manuscrito aceptado: Enero 16, 2017. 


\section{Introducción}

La Formación San Cassiano del Ladiniano tardío - Carniano medio (región de las Dolomitas, NE de Italia) ha sido ampliamente conocida y estudiada por su diversidad de fauna y el estado de conservación de la misma, especialmente la proveniente de las localidades de Alpe di Specie y Misurina (Fürsich y Wendt, 1977; Sánchez-Beristain y Reitner, 2016). Esta unidad litológica porta su nombre a partir del poblado de San Cassiano, en la región autónoma de Alto Adigio (Bosellini et al., 2005; Figura 1). Consiste en depósitos de cuenca (intercalaciones de marga y turbiditas de origen volcánico) situados entre arrecifes masivos y áreas post-arrecifales locales, todos ellos depositados a su vez de manera concordante sobre la Formación Wengen de edad ladiniana, consistente en intercalaciones de lutita y material vulcanoclástico (Wendt y Fürsich, 1980).
La Formación San Cassiano consta de dos miembros: un Miembro Inferior (de edad ladiniano-carniana) y un Miembro Superior, de edad plenamente carniana. Ambos miembros afloran de manera representativa en la localidad de Alpe di Specie (Figura 2), con una litología muy similar, presentando estratos constituidos por calizas y margas intercaladas. Estos estratos son ricos en fósiles (Fürsich y Wendt, 1977; Reitner, 1987; Russo et al., 1997). La preservación de éstos es extraordinaria, lo cual es constatable porque gran parte de ellos conserva su mineralogía original (Neuweiler y Reitner, 1995; Sánchez-Beristain y Reitner, 2012), al grado de que ciertas localidades, donde esta formación aflora, son consideradas como $\mathrm{La}$ gerstätten fósiles (Fürsich, 2000).

Si bien el grado de preservación es alto en los depósitos de la Formación San Cassiano, lo es aún mayor en los olistolitos denominados Cipit, conocidos comúnmente como Cipit boulders en la litera-

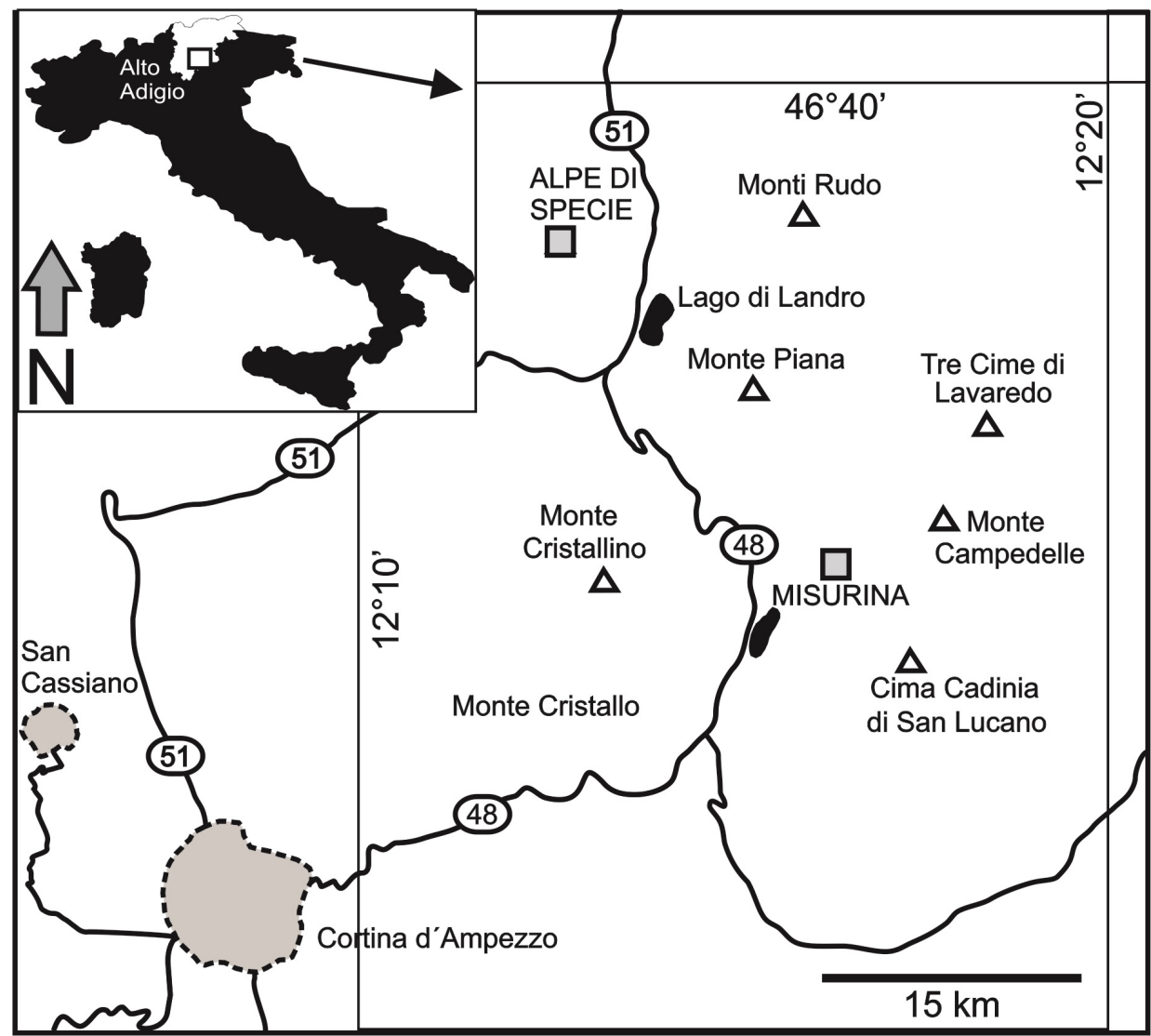

Figura 1 Ubicación de las dos localidades (Alpe di Specie y Misurina) en el área de estudio en la región de las Dolomitas, NE de Italia. 
tura en lengua inglesa, y cuya etimología a su vez proviene del topónimo modificado del arroyo Tschapit (von Richthofen, 1860). Estas rocas se derivan, en su mayoría, de los parches arrecifales (patch reefs, e.g. Wendt, 1982) y de las facies de plataforma (mayormente dolomitizadas), que fueron sujetas a procesos de emersión y karstificación posterior (Biddle, 1981; Russo et al., 1997). Dichos procesos propiciaron el asentamiento de estos olistolitos desde los márgenes de la plataforma hacia la cuenca casiana (Russo et al., 1997; Sánchez-Beristain, 2010). Los olistolitos proceden en su totalidad del Miembro Superior de la Formación San Cassiano (Figura 2), y presentan microbialitas (hasta en un $75 \%$ ), alomicritas (hasta en un $50 \%$ ), componentes esqueléticos constituyendo framestones (hasta en un $50 \%$ ) y cementos (hasta en un $10 \%$ ) como facies dominantes. Normalmente, las facies microbialíticas y las framestones son mutuamente excluyentes, o bien, ante la dominancia de una, la otra tiende a disminuir considerablemente (Rech, 1998; Sánchez-Beristain y Reitner, 2016). Para mayores detalles en cuanto a la estratigrafía de la región, se refiere a los lectores al trabajo de Müller-Wille y Reitner (1993).

Como parte de las framestones de los olistolitos Cipit, las esponjas destacan al ser el componente dominante (Reitner, 1987; Russo, 2005; Sánchez-Beristain y Reitner, 2016). Otros taxones constructores, como los corales, no tienen una relevancia considerable en estas facies (Fürsich y Wendt, 1977; Hausmann y Nützel, 2015). Lo mismo aplica para los briozoos, de los cuales, si bien existe una diversidad considerable para la Formación San Cassiano, no representan una fracción importante (Fürsich y Wendt, 1977; Russo, 2005; Nützel y Kaim, 2014). Sin embargo, una excepción la constituye la especie Reptonoditrypa cautica Schäfer y Fois, la cual desempeñó un papel trascendental como incrustante dentro de una asociación particular con la esponja talámida Cryptocoelia zitteli Steinmann.

En este trabajo se revisará por primera vez su rol paleoecológico a detalle.

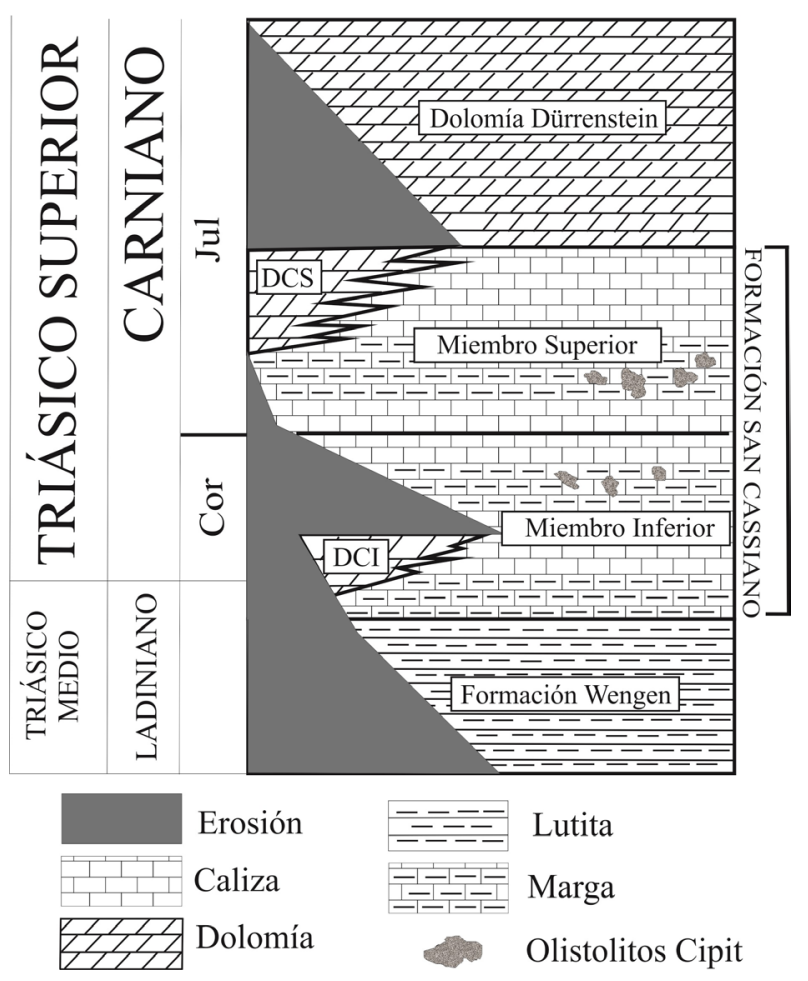

Figura 2 Sección estratigráfica de la Formación San Cassiano (Triásico Medio - Superior) en las localidades Alpe di Specie. DCI = Dolomía Casiana Inferior. DCS = Dolomía Casiana Superior. Cor = Cordevoliano. Jul = Juliano. Ver texto y referencias en el mismo para mayor detalle. 


\section{2. Área de estudio}

El área de trabajo de donde procede el material se encuentra en dos localidades de la región de las Dolomitas, en el Tirol del Sur, NE de Italia. La localidad de origen para la mayoría de las muestras es Alpe di Specie (46 38' 29" N, $12^{\circ} 11^{\prime} 44^{\prime \prime}$ E), célebre por la preservación de las muestras que se encuentran en ella (Russo et al., 1997). La otra localidad es Misurina, en Valle di Rimbianco $\left(46^{\circ}\right.$ 35' 0" N; $12^{\circ} 15^{\prime} 89^{\prime \prime}$ E). El Miembro Superior de la Formación San Cassiano (Figura 2) aflora en las dos localidades mencionadas.

\section{Materiales y métodos}

Setenta y siete olistolitos fueron colectados. Se obtuvieron varias láminas delgadas de cada uno de ellos (como mínimo una y como máximo ocho; en total 398 láminas en formato 10 x $15 \mathrm{~cm}$ y 7.5 x 10 $\mathrm{cm})$, a modo de determinar su estado de preservación vía microscopía óptica con ayuda de la técnica de tinción de carbonatos de Dickson (1965), y con ello poder llevar a cabo de manera efectiva la identificación taxonómica. De estas 398 láminas, 96 constituyen remanentes de facies arrecifales y fueron sujetas al análisis mencionado, 54 láminas proceden de la localidad Alpe di Specie, y 42 de la localidad de Misurina.

El material está depositado en la Colección de Paleontología del Centro de Geociencias de la Universidad de Göttingen (GZG), en Alemania.

\section{Resultados}

De las 96 láminas analizadas, el briozoo Reptonoditrypa cautica Schäfer y Fois fue encontrado en 37 de ellas, 21 de las mismas proceden de Alpe di Specie, y 16 de Misurina. Se analizan a detalle las implicaciones paleoecológicas que este taxón presenta.

\subsection{PALEONTOLOGÍA SISTEMÁTICA}

Reino Animalia Linnaeus, 1758

Phylum Bryozoa Ehrenberg, 1831

Clase Stenolaemata Borg, 1926

Orden Trepostomata Ulrich 1882

Familia Heterotrypidae Ulrich, 1890

Género Reptonoditrypa Schäfer y Fois, 1987

Reptonoditrypa cautica Schäfer y Fois, 1987

\subsubsection{SINONIMIA}

1987. Reptonoditrypa cautica n.sp. Schäfer y Fois, p.

184-186; fig. 2; lámina 7, figs. 1-4; lámina 8, figs. 1-4.

1993. Reptonoditrypa cautica Schäfer y Fois, Senow

bari-Daryan et al., p. 182, 194, 198, 218,

226, 242, 246; láminas 44-7; 46-2,5; 47-1,2,3;

48-1,3,6-7; 49,2-4; 50-7; 53-1.

2003. Reptonoditrypa cautica Schäfer y Fois. Schäfer et al., p. 144, 146.

2004. Reptonoditrypa cautica Schäfer y Fois, Flügel,

p. 516-517; lámina 85, fig. 1 .

2005. Reptonoditrypa cautica Emmerich et al., p.

580-581; 583-584, 587, 599; lámina 8, figs.

5-6; lámina 10, fig. 12.

2006. Reptonoditrypa cautica Schäfer y Fois, Enos $e t$ al., p. 53.

2008. Reptonoditrypa cautica Schäfer y Fois, Powers y Pachut, p. 366.

2016. Reptonoditrypa cautica Sánchez-Beristain y Reitner, p. 255, 260, 266; figs. 11e, 20a.

\subsubsection{DIAGNOSIS ORIGINAL (SCHÄFER Y FOIS, 1987)}

Zoarios de planos a columnares, compuestos de numerosas subcolonias hemisféricas separadas por una extensión de la pared basal; las nuevas subcolonias se originan de autozoecios preexistentes ubicados en el centro de la subcolonia, persistentes de la generación subyacente. La endozona de cada subcolonia es estrecha, restringida a la región inmediatamente superior a la pared basal. Consiste en autozoecios horizontales que rápidamente se 
pliegan hacia arriba. La gemación de los autozoecios en cada subcolonia se da a partir de la pared basal y de forma acíclica a partir de los vértices de los zoecios remanentes de generaciones anteriores. La exozona de cada subcolonia se compone de autozoecios que aparecen subcirculares en sección tangencial, con diafragmas basales estrechamente espaciados y una cámara habitacional pequeña. Las paredes de los zoecios están ligera y uniformemente engrosadas. Mesozoecios abundantes, formando un anillo alrededor de cada autozoecio, subcirculares en sección tangencial, con diafragmas igualmente distribuidos que los autozoecios. Acantostilos pequeños, comunes, irregularmente dispersos en las paredes de los zoecios. Microestructura lamelar convexa en las paredes verticales. Las paredes basales son de color amarillento sin una microestructura visible.

\subsubsection{DESCRIPCIÓN}

Zoarios con un hábito de crecimiento plano. El ancho de estas colonias varía de 5.5 y $8 \mathrm{~mm}$, y están conformados por una capa sencilla. El diámetro interno de los autozoecios oscila entre 0.16 y $0.19 \mathrm{~mm}$ (Figuras 3a-b). Ocasionalmente, los autozoecios pueden exhibir una forma poligonal redondeada (Figuras 3b-d; 4a-b), delimitada por los mesozoecios, que son comunes y circulares, y se disponen a su vez de forma anular alrededor de los autozoecios (Figura 3b, d). Acantostilos presentes en las paredes de los zoecios, y visibles en sección longitudinal (Figuras 4c-d). No se observaron detalles de la microestructura. La tinción de Dickson (1965) refleja una mineralogía de calcita alta en magnesio, determinada por la coloración rosácea de las colonias (Figuras 3-5).

\subsubsection{DISCUSIÓN}

Los ejemplares encontrados en los depósitos de la Formación San Cassiano muestran únicamente zoarios aplanados (Figuras $4 \mathrm{c}-\mathrm{d}$; 5a-b), concordando en parte con la diagnosis original de Schäfer y Fois (1987), en donde establecen que pueden ser también columnares. Asimismo, es importante
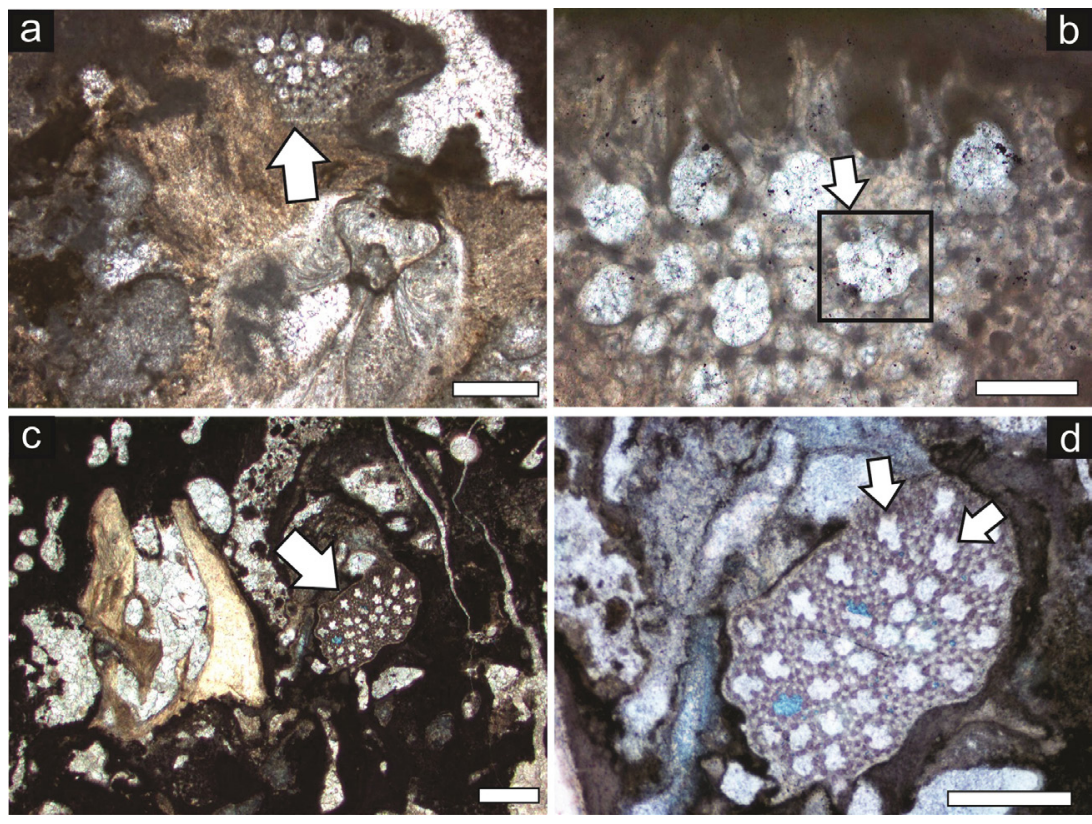

Figura 3 a) Sección transversal de una colonia de Reptonoditrypa cautica Schäfer y Fois (flecha) encostrada sobre un bioclasto. Muestra GZG JR III-53. Escala $=800 \mu \mathrm{m}$. b) Magnificación de a). Nótese el contorno con tendencia poligonal del autozoecio (flecha y recuadro) y la disposición anular de los mesozoecios a su alrededor. Escala $=200 \mu \mathrm{m}$. c) Sección transversal de una colonia de Reptonoditrypa cautica Schäfer y Fois (flecha), como parte del gremio de los encostrantes, en conjunto con el alga coralinácea Mesophyllum. Nótese el bioclasto (color amarillo-anaranjado) Muestra GZG JR III-A1. Escala $=800 \mu \mathrm{m}$. d) Magnificación de c). Nótense los contornos de los zoecios. Escala $=800 \mu \mathrm{m}$. 


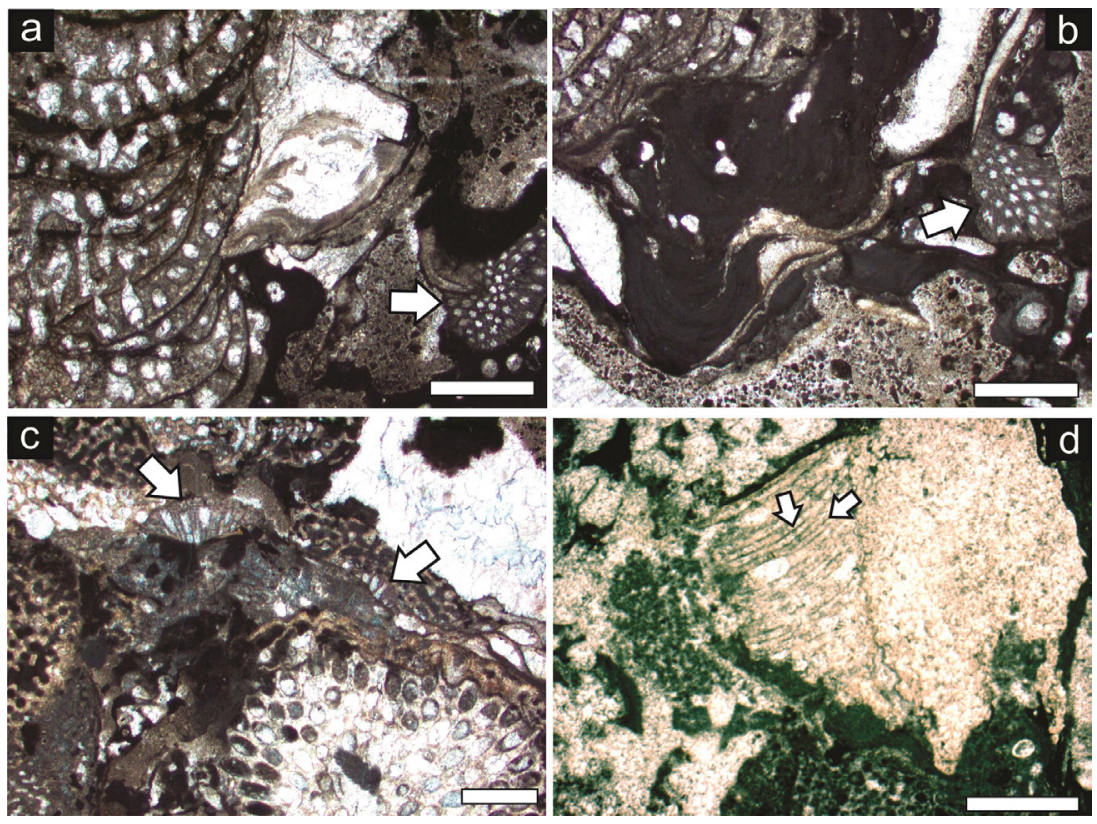

Figura 4 a) Sección transversal de una colonia de Reptonoditrypa cautica Schäfer y Fois (flecha) encostrada dentro del alga Mesophyllum. A la izquierda, la esponja talámida Cryptocoelia zitteli. Muestra GZG JR III-46. Escala = $2 \mathrm{~mm}$. b) Sección oblicua de R. cautica (flecha). Muestra GZG JR III-46. Escala = $2 \mathrm{~mm}$. c) Sección longitudinal de dos colonias de R. cautica (flechas) en la asociación Amblisyphonella-Megalodon, dominada por esponjas. Nótese el efecto unificador/encostrante de las colonias, fusionándose con los constructores a su alrededor (sobrelapamiento de gremios). Muestra GZG JR III-45. Escala $=1 \mathrm{~mm}$. d) Sección longitudinal de $R$. cautica. Nótense los acantostilos abundantes (flechas). Muestra GZG JR III-B352. Escala = $850 \mu \mathrm{m}$.
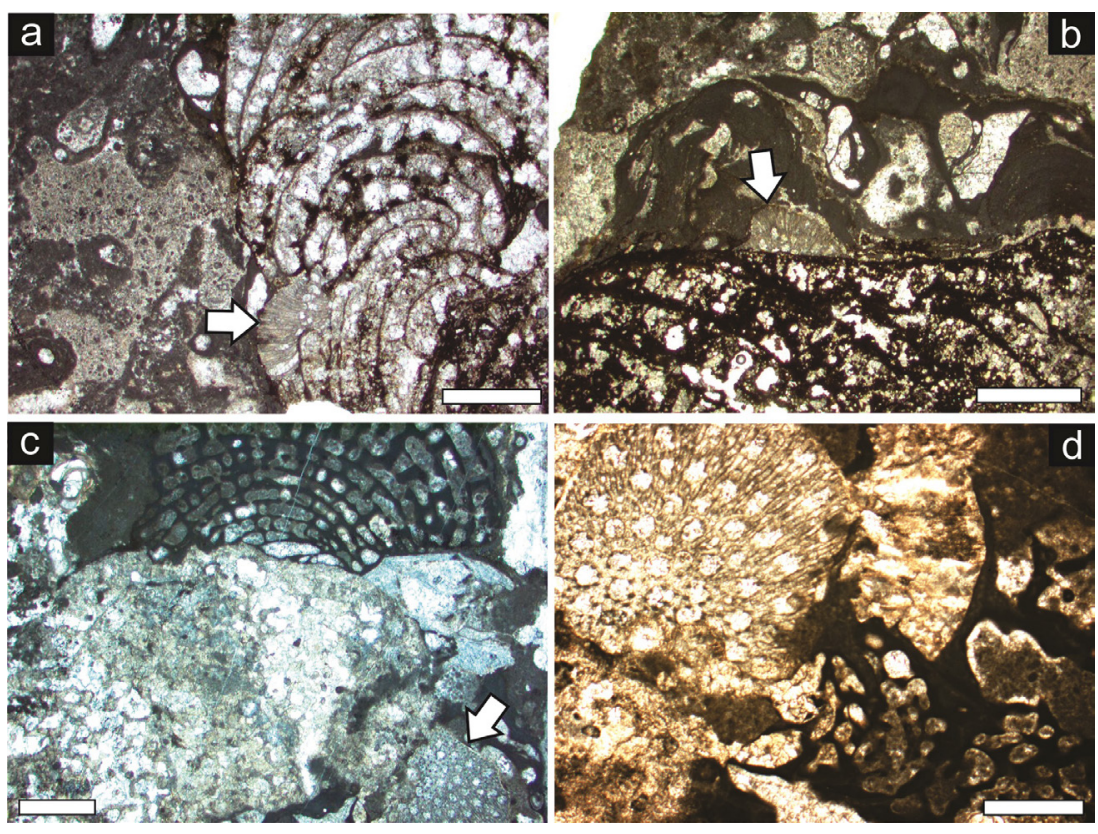

Figura 5 a) Sección longitudinal de una colonia de Reptonoditrypa cautica Schäfer y Fois (flecha), encostrada sobre las cámaras de la esponja talámida Cryptocoelia zitteli (derecha). Muestra GZG JR III-B347. Escala $=1 \mathrm{~mm}$. b) Sección longitudinal de $R$. cautica (flecha) sobre otro ejemplar de $C$. zitteli. El briozoo es, a su vez, encostrado por el alga Mesophyllum. Muestra GZG JR III-58. Escala = 1.5 mm. c) Colonia de R. cautica (flecha) en la Asociación Cassianothalamia (e.g. Keupp et al., 1989). La esponja talámida Cassianothalamia zardinii (centro, arriba) es el elemento más distintivo. Muestra GZG CGI-10. Escala $=1.5 \mathrm{~mm}$. d) Colonia de $R$. cautica (esquina superior izquierda) siendo encostrada por C. zardinii (derecha). Muestra GZG JR II-2. Escala $=800 \mu \mathrm{m}$. Ver texto para mayores detalles. cautica. Nótense los acantostilos abundantes (flechas). Muestra GZG JR III-B352. Escala = $850 \mu \mathrm{m}$. 
resaltar la forma poligonal redondeada de los autozoecios en corte transversal (Figuras 3b, d), un rasgo que no fue originalmente descrito en 1987. Adicionalmente, se debe puntualizar que el alcance estratigráfico inicialmente asignado a Reptonoditrypa cautica en su descripción original (Anisiano) se extiende con el referido en el presente trabajo (Ladiniano-Carniano), tomando como base la edad asignada a los olistolitos Cipit (e.g. Reitner, 1987; Bosellini, 1991; Müller-Wille y Reitner, 1993, Sánchez-Beristain y Reitner, 2016).

Por otra parte, existen similitudes entre estos hallazgos y la especie Reptonoditrypa abadhenensis del Triásico Tardío (Noriano) de Irán, esencialmente a nivel de la distribución anular de los mesozoecios alrededor de los autozoecios (Figuras 3b, d, comparar Schäfer et al., 2003). Sin embargo, el diámetro interno de los autozoecios de los organismos analizados en este trabajo (0.169 a 0.192 mm, Figura 3b) es, por lo general, mayor y con una mayor desviación estándar que los de la especie del Noriano/Rhaetiano del Medio Oriente (Schäfer et al., 2003; lámina 28, figs. 2-3), en concordancia con la descripción original de $R$. cautica (0.158 a $0.187 \mathrm{~mm}$ ) por parte de Schäfer y Fois (1987).

No obstante, a pesar de las diferencias de rangos estratigráficos expuestos en la descripción original de $R$. cautica (Schäfer y Fois, 1987) y del propio de $R$. abadhenensis (Schäfer et al., 2003), así como de las diferencias morfológicas principalmente a nivel autozoecio y forma del crecimiento del zoario, no consideramos que los rasgos descritos para el material de la Formación San Cassiano sean suficientes para asignar el material a una nueva especie, debido principalmente a la escasez de ejemplares que muestran caracteres diferentes a los reportados en la diagnosis original.

\subsection{PALEOEGOLOGÍA DE REPTONODITRYPA CAUTICA}

Reptonoditrypa cautica Schäfer y Fois es una especie de briozoo con hábito de incrustante en la Formación San Cassiano; esto es, se desarrolla fijándose al sustrato de forma que su expansión sea lo más amplia posible en la comunidad arrecifal en detrimento de su espesor. Este tipo de crecimiento se encuentra normalmente asociado a ambientes de alta energía (Smith, 1995; Taylor y Wilson, 2003; Taylor y Waeschenbach, 2015). Para comprender la función de $R$. cautica en los depósitos arrecifales de la Formación San Cassiano (Wendt, 1982), se utilizó el modelo de Fagerstrom (1987, 1991), el cual, si bien ha recibido algunas críticas (e.g. Precht, 1994), también ha sido ampliamente aceptado (e.g. Grötsch, 1994; Webb, 2001; Rasser y Riegl, 2002; Bush et al., 2007; Lombardi et al., 2014). Este modelo contempla la función de cada organismo en este tipo de ecosistemas a lo largo del tiempo geológico, y es aplicable al estudio de los arrecifes fósiles y recientes. A diferencia del concepto que existe sobre los arrecifes desde un punto de vista geológico, en el cual son considerados principalmente como formaciones y estructuras dinámicas en un contexto sedimentario, en el modelo de Fagerstrom se los contempla como comunidades o remanentes de las mismas, principalmente desde un punto de vista ecológico. Este modelo se fundamenta en el concepto de gremio (guild), acuñado por Root (1967). Un gremio es definido como un grupo de especies que explotan la misma clase de recursos ambientales de una forma similar, sin importar la taxonomía de sus integrantes. Por esta razón, los requerimientos de nicho de estas especies pueden traslaparse de manera significativa. Con ello, una especie puede pertenecer a más de un gremio. Este concepto da un énfasis especial a todas las especies distribuidas simpátricamente e involucradas en una interacción del tipo competencia (Townsend et al., 2008). La propuesta de Fagerstrom propone una clasificación de los organismos que habitan o habitaron los arrecifes de acuerdo a la función que desempeñen dentro de su gremio; es decir, el rol ecológico, estructural y composicional que llevan a cabo en el desarrollo, mantenimiento y evolución de estos ecosistemas. Fagerstrom $(1987,1991)$ propuso que el tipo y 
orientación de sustrato, tomando en cuenta la forma en la que sean aprovechados los recursos, puede definir las categorías gremiales, lo cual en esencia vendría siendo una variante del concepto de "nicho ecológico" de Grinnell (1917) o del de "ecoespacio" de Bambach et al. (2007). Con estos antecedentes, se puede concluir a priori que debería existir una mayor similitud morfológica dentro de los integrantes de un mismo gremio que entre integrantes de distintos gremios; y, que cada gremio lleva a cabo una función particular dentro del desarrollo exitoso de los ecosistemas arrecifales. Considerando esta premisa, los organismos constructores (constructors) con su masivo crecimiento en dirección vertical y lateral proveen de una especie de andamiaje que sirve de soporte para la estructura inicial, además de constituir la mayor parte de su volumen. Los amortiguadores (bafflers), por su parte, actúan como una barrera laxa, disminuyendo la velocidad de las corrientes. Esto conlleva de manera inmediata a la posibilidad de que se depositen las partículas sedimentarias. El crecimiento en todos sentidos de los unificadores o incrustantes (binders) incorpora los sedimentos producidos por el mismo arrecife, así como también a los propios organismos, consolidando la rigidez de la estructura. Los destructores (destroyers) reducen el crecimiento desmedido de la estructura a través de la acción erosiva que desempeñan, y, por último, los moradores (dwellers) ejercen un rol mayormente pasivo, y cuya presencia en el ecosistema arrecifal se debe a que pueden obtener recursos del mismo, como alimento, refugio o protección dentro de la estructura sólida.

El medio preferido de fijación de Reptonoditrypa cautica es la esponja Cryptocoelia zitteli y las inmediaciones donde ésta se desenvuelve (Figuras $4 \mathrm{a}-\mathrm{b}$; 5a-b). Esta esponja talámida, por su forma de crecimiento, pertenecería al gremio de los amortiguadores (comparar Bergquist, 1978; Villamizar y Laughlin, 1992; Carrera, 2000). Reptonoditrypa se desarrolla en los resquicios intercamerales de la esponja, donde encuentra su sustrato, contribuyendo a la consolidación de la rigidez de la estructura
(Figuras 5a-b). Una asociación similar esponja-briozoo incrustante fue observada por Carrera (2000) en la Formación San Juan del Llanvirn temprano (Ordovícico Medio) de Argentina, en donde se describió un briozoo cyclostomátido similar al género Berenicea incrustando a la esponja litístida Patellispongia.

Adicionalmente, $R$. cautica puede ser encontrado sobre -y dentro de- parches del alga coralinácea Mesophyllum (Figuras 3c-d; 4a-b), la cual, al incrustarse directamente sobre $C$. zitteli, formaría parte del gremio de los incrustantes/constructores. Es posible incluso detectar zonas en las cuales $R$. cautica desempeña el papel de nexo entre $C$. zitteli y Mesophyllum, reforzándose así su rol como incrustante (Figura 5b). Este tipo de asociaciones también puede encontrarse en la actualidad. Por ejemplo, Rosso et al. (2010) informan sobre una especie nueva de briozoo queilostómido, Bertorsonidra prenanti, con un modo de vida incrustante sobre algas coralináceas que habitan en las costas de Sicilia, Italia. En nuestro estudio, briozoos y algas se desempeñan como actores en la captación de bioclastos, promoviendo el desarrollo del arrecife (Figura 3c). Un hallazgo interesante es que Cryptocoelia zitteli está siempre vinculada a la presencia de Reptonoditrypa cautica. Por el contrario, en las láminas donde aparece el briozoo, no siempre es posible encontrar a la esponja.

Existe otro tipo de asociaciones en los olistolitos de la Formación San Cassiano, como es el caso de la Asociación Cassianothalamia (Keupp et al., 1989; Sánchez-Beristain y Reitner, 2016), caracterizada por la presencia de organismos típicos de un ambiente críptico, como la esponja talámida Cassianothalamia zardinii, y por la ausencia total de algas. La presencia de ciertos microincrustantes como Terebella lapilloides y Tubiphytes obscurus (ambos moradores/destructores) vistos en las mismas láminas delgadas refuerzan, además, la predominancia de condiciones de baja energía, típicas de altas profundidades. La presencia de $R$. cautica en asociaciones provenientes de ambientes donde predominan condiciones de baja energía (Figuras $5 \mathrm{c}^{-} \mathrm{d}$ ), podría 
ser indicadora de un aumento esporádico en las condiciones de la misma.

Si tenemos en cuenta que (1) en la Asociación Amblisyphonella-Megalodon constituida principalmente por esponjas (Sánchez-Beristain, 2010), el abundante incrustamiento de $R$. cautica es un caso particular en el que el briozoo se incrusta sobre microbialitas que toman a su vez a los constructores como sustrato (Figura 4c), formando un macizo sólido que resistiría frente al oleaje (Fürsich y Wendt, 1977; Zell, 2014); y (2) que los olistolitos Cipit son remanentes de los Patch Reefs que Wendt (1982) definió por primera vez, se podría interpretar que $R$. cautica forma parte de un sobrelapamiento de gremios en la Formación San Cassiano, en particular con el gremio de los amortiguadores, el de los constructores, y el de los encostrantes/ unificadores.

\section{Conclusiones}

Reptonoditrypa cautica Schäfer y Fois es un briozoo que fue parte activa del gremio de los organismos incrustantes en los ecosistemas arrecifales del Triásico Medio-Tardío en la Formación San Cassiano. Este briozoo sólo se encuentra en su morfotipo aplanado dentro de los olistolitos Cipit, lo cual facilita su ubicación dentro del gremio de los incrustantes.

Normalmente se encuentra como parte de asociaciones donde las esponjas, como es el caso de Cryptocoelia zitteli, Cassianothalamia zardinii y Amblisyphonella strobiliformis, son el componente predominante. Es de destacar su nexo con costras del alga coralinácea Mesophyllum, y el hecho de que tenga la capacidad de unificar clastos, costras microbianas y otros organismos dentro de la asociación fósil, lo que implica un sobrelapamiento de gremios entre los incrustantes, los constructores y los amortiguadores.

\section{Agradecimientos}

Los autores desean agradecer a las autoridades de la Facultad de Ciencias, UNAM (Ciudad de México) y al Courant Centre de Geobiología (Centro de Geociencias, Universidad de Göttingen, Alemania) por el constante apoyo recibido para la realización de sus investigaciones. De la misma manera, agradecemos sinceramente a la Dra. Consuelo Sendino (Departamento de Ciencias de la Tierra, Museo de Historia Natural, Reino Unido), así como también a la M. en G. Itzia E. Nieto López, a la M. en C. Laura López Esquivel K., y al M. en C. Sergio González-Mora (Facultad de Ciencias, UNAM) por haber proporcionado literatura de dificil acceso para los autores.

Asimismo, se extiende el agradecimiento al Dr. Miguel Ángel Torres Martínez (Instituto de Geología, UNAM), y a un revisor anónimo, cuyos atinados comentarios y sugerencias ayudaron a mejorar de manera sustancial la calidad de este manuscrito.

\section{Dedicatoria}

El presente manuscrito está dedicado a la memoria de María Estela Beristain Sánchez (19432016), devota madre, esposa, abuela, y, sobre todo, amiga.

\section{Referencias}

Bambach, R.K., Bush, A.M., Erwin, D.H., 2007, Autecology and the filling of ecospace: key metazoan radiations: Palaeontology, 50, $1-22$.

Bergquist, P.R., 1978, Sponges: London, Hutchinson, $276 \mathrm{p}$. 
Biddle, K.T., 1981, The basinal Cipit boulders: indicators of Middle to Upper Triassic buildup margins, Dolomite Alps, Italy: Rivista Italiana di Paleontologia e Stratigrafia, 86, 779-794.

Borg, F., 1926, Studies on recent cyclostomatous Bryozoa: Zoologiska Bidrag från Uppsala, 10, 181-507.

Bosellini, A., 1991, Geologia delle Dolomiti: Bolzano, Casa Editrice Ahesia, 192 p.

Bosellini, A., Gianolla, P., Stefani, M., 2005, Geology of the Dolomites: Episodes, 26(3), 181-185.

Bush, A.M., Bambach, R.K., Daley, G.M., 2007, Changes in theoretical ecospace utilization in marine fossil assemblages between the midPaleozoic and late Cenozoic: Paleobiology, 33(1), 76-97.

Carrera, M.G., 2000, Epizoan-sponge interactions in the Early Ordovician of the Argentine Precordillera: Palaios, 15, 261-272.

Dickson, J.A.D., 1965, A modified staining technique for carbonates in thin section: Nature, 205, 587.

Ehrenberg, G.G., 1831, Symbolae physicae. Animalia Evertebrata exclusis insectis. Series prima cum tabularum decade prima: Berlin, Mittler, 135 p.

Emmerich, A., Zamparelli, V., Bechstädt, T., Zuehlke, R., 2005, The reefal margin slope of a Middle Triassic carbonate platform: The Latemar (Dolomites, Italy): Facies, 50(3), 573-614.

Enos, P., Lehrmann, D.J., Jiayong, W., Youyi, Y., Jiafei, X., Minzoni, D.H., Berry, A.K., Montgomery, P., 2006, Yangtze Platform in Guizhou Province, People's Republic of China: Geological Society of America Special Paper, 47, 1-105.

Fagerstrom, J.A., 1987, The evolution of reef communities: New York, John Wiley \& Sons, $600 \mathrm{p}$.

Fagerstrom, J.A., 1991, Reef-bulding guilds and a checklist for determining guild membership. A new approach for study of communities: Coral Reefs, 10, 47-52.

Flügel, E., 2004, Microfacies of carbonate rocks. Analysis, interpretation and application: Berlin-Heidelberg, Springer, 984 p.

Fürsich, F.T., 2000, Die Cassianer Schichten, in Meischner, D., Pinna, G. (eds.), Europäische Fossillagerstätte: Berlin Heidelberg, Springer, 79-82.

Fürsich, F.T., Wendt, J., 1977, Biostratinomy and Palaeoecology of the Cassian Formation (Triassic) of the Southern Alps: Paleogeography Palaeoclimatology Palaeoecology, 22, 257-323.

Grinnell, J., 1917, The niche relationships of the California thrasher: The Auk, 34, 427-433.

Grötsch, J., 1994, Guilds, cycles and episodic vertical aggradation of a reef (Late Barremian to Early Aptian, Dinaric carbonate platform, Slovenia), in DeBoer, P.L., Smith, D.G. (eds.), Orbital forcing and cyclic sequences: Special Publications of the International Association of Sedimentologists, 19, 227-242.

Hausmann, I.M., Nützel, A., 2015, Diversity and palaeoecology of a highly diverse Late Triassic marine biota from the Cassian Formation of north Italy: Lethaia, 48(2), 235-255.

Keupp, H., Reitner, J., Salomon, D., 1989, Kieselschwämme (Hexactinellida und "Lithistida") aus den Cipit-Kalken der Cassianer Schichten (Karn, Südtirol): Berliner geowissenschaftliche Abhandlungen, A 106, 221-241.

Linnaeus, C., 1758, Systema Naturae, I: Holmiae, Laurentii Salvii, 824 p.

Lombardi, C., Taylor, P.D., Cocito, S., 2014, Bryozoan constructions in a changing Mediterranean Sea, in Goffredo, S., Dubinsky, Z. (eds.), The Mediteranean Sea: Its history and present challenges: Dordrecht, Springer, 373-384 
Müller-Wille, S., Reitner, J., 1993, Palaeobiological Recontructions of selected sphinctozoan sponges from the Cassian Beds (Lower Carnian) of the Dolomites (Northern Italy): Berliner geowissenschaftliche Abhandlungen, E 9, 253-281.

Neuweiler, F., Reitner, J., 1995, Epifluorescencemicroscopy of selected automicrites from lower Carnian Cipit-boulders of the Cassian Formation (Seeland Alpe, Dolomites): Facies, $32,26-28$.

Nützel, A., Kaim, A., 2014, Diversity, palaeoecology and systematics of a marine fossil assemblage from the Late Triassic Cassian Formation at Settsass Scharte, N Italy: Paläontologische Zeitschrift, 88, 405-431.

Powers, C.M., Pachut, J.E., 2008, Diversity and distribution of Triassic bryozoans in the aftermath of the end-Permian mass extinction: Journal of Paleontology, 82(2), 362-371.

Precht, W., 1994, The use of the term guild in coral reef ecology and paleoecology: a critical evaluation: Coral Reefs, 13, 135-136.

Rasser, M.W., Riegl, B., 2002, Holocene coral reef rubble and its binding agents: Coral Reefs, $21,57-72$.

Rech, H., 1998, Geobiologie der sogenannten "Cipit-Kalke" der Beckenfazies der Cassianer-Schcihten, S'St. Kassian, Dolomiten: Göttingen, Alemania, GeorgAugust Universität Göttingen, tesis de maestría, $136 \mathrm{p}$.

Reitner, J., 1987, A new calcitic sphinctozoan sponge belonging to the Demospongiae from

the Cassian Formation(Lower Carnian; Dolomites, Northern Italy) and its phylogenetic

relationship: Geobios, 20, 571-589.

Root, R.B., 1967, The niche exploitation pattern in the blue-gray gnatcatcher: Ecological Monographs, 97, 317-350.

Rosso, A., Sciuto, F., Sinagra, A., 2010, Bertorsonidra n.gen. (Bryozoa,
Cheilostomata) for the Tremopora prenanti Gautier, 1955, a rare species from the Mediterranean: Zoosystema, 32(3), 457-467.

Russo, F., 2005, Biofacies evolution in the Triassic platforms of the Dolomites, Italy: Annali dell' Università degli Studi di Ferrara, Museologia Scientifica e Naturalistica, Volume Speciale 2005, 33-45.

Russo, F., Neri, C., Mastandrea, A., Baracca, A., 1997, The Mud Mound Nature of the Cassian Platform Margins of the Dolomites. A Case History: the Cipit Boulders from Punta Grohmann (Sasso Piatto Massif, Northern Italy): Facies, 36, 25-36.

Sánchez-Beristain, J.F., 2010, Paleoecological and geochemical studies on sponge/ microencruster -bearing communities contained in selected Cipit Boulders from the St. Cassian Formation (Lower Carnian, Upper Triassic) of the Dolomites, northeastern Italy: Göttingen, Alemania, Georg-August Universität Göttingen, tesis doctoral, $146 \mathrm{p}$.

Sánchez-Beristain, F., Reitner, J., 2012, Paleoecology of microencrusters and encrusting "coralline" sponges in Cipit boulders from the Cassian Formation (upper Ladinian-lower Carnian, Dolomites, Northern Italy): Paläntogische Zeitschrift, 86, 113-133.

Sánchez-Beristain, F., Reitner, J., 2016, Palaeoecology of new fossil associations from the Cipit boulders, St. Cassian Formation (Ladinian-Carnian, Middle-Upper Triassic; Dolomites, NE Italy): Paläontologische Zeitschrift, 90(2), 243-269.

Schäfer, P., Fois, E., 1987, Systematics and evolution of Triassic Bryozoa: Geologica et Palaeontologica, 21, 173-225.

Schäfer, P., Sebowbari-Daryan, B., Hamedani, A., 2003, Stemolaemate bryozoans from the Upper Triassic (Norian-Rhaetian) Nayband Formation, Central Iran: Facies, 49, 135-150. 
Senowbari-Daryan, B., Zühlke, R., Bechstädt, T., Flügel, E., 1993, Anisian (Middle Triassic) buildups of the Northern Dolomites (Italy): The recovery of reef communities after the Permian/Triassic crisis: Facies, 28, 181-256.

Smith, A.M., 1995, Palaeoenvironmental interpretation using bryozoans: A review, in Bosence, D.W.J., Allison, P.A. (eds.), Marine Palaeoenvironmental Analysis from Fossils: Geological Society of London, Special Publications, 83, 231-243.

Taylor, P.D., Waeschenbach, A., 2015, Phylogeny and diversification of bryozoans: Palaeontology, 58(4), 585-599.

Taylor, P.D., Wilson, M.A., 2003, Palaeoecology and evolution of marine hard substrate communities: Earth-Science Reviews, 61, $1-103$.

Townsend, C.R., Begon, M., Harper, J.L., 2008, Essentials of ecology: New York, WileyBlackwell, $532 \mathrm{p}$.

Ulrich, E.O., 1882, American Palaeozoic Bryozoa: Journal of the Cincinnati Society of Natural History, 5, 232-257.

Ulrich, E.O., 1890, Part II. Palaeontology of Illinois. Section VI. Palaeozoic Bryozoa:

Report of the Geological Survey of Illinois, 8, 283-688.
Villamizar, E., Laughlin, R.A., 1992, Fauna associated with the sponges Aplysina archeri and Aplysina lacunosa in a coral reef of the Archipiélago de los Roques, National Park, Venezuela, in Reitner, J., Keupp, H. (eds.), Fossil and recent sponges: New York, Springer, 522-542.

Von Richthofen, F., 1860, Geognostische Beschreibung der Umgegend von Pedrazzo, St. Cassian und der Seiser Alpe in Südtirol: Gotha, Justus Perthes, 327 p.

Webb, G.E., 2001, Biologically induced carbonate precipitation in reefs through time, in Stanley, G.D. Jr. (ed.), The history and sedimentology of ancient reef systems: New York: Kluwer Academic/Plenum Publishers, 159-203.

Wendt, J., 1982, The Cassian Patch Reefs (Lower Carnian, Southern Alps): Facies, 6, 185-202.

Wendt, J., Fürsich, F.T., 1980, Facies analysis and paleogeography of the Cassian Formation, Triassic, Southern Alps: Rivista Italiana di Paleontologia e Stratigrafia, 85(3/4), 1003-1028.

Zell, L., 2014, The Great Barrier Reef: A Journey through the world's greatest natural wonder: Crows Nest, Murdoch Books, 250 p. 